\title{
Analysis and Improvement on Robustness of AQM in DiffServ Networks
}

\author{
Wei Liu, Jianhua He, Zongkai Yang \\ Department of Electronics and Information Engineering \\ Huazhong University of Science and Technology \\ Wuhan 430074, P.R.China
}

\begin{abstract}
The queue performance in delay and loss of RIO suffers in parameter setting sensitivity to the traffic load and user-defined subscription. We analyze the steady queue length of RIO and investigate its changing principles under different traffic load. We observed that, it is necessary to dynamically adjust the control policy of RIO algorithm to keep the queue in stable state. Two adaptive versions of RIO algorithm are proposed to solve this problem for delay-sensitive and loss-sensitive application respectively. Simulation results show that these two enhanced RIO algorithms can effectively improve the robustness of RIO algorithm on the performance of delay or loss under the change of traffic load and user-defined subscription.
\end{abstract}

Key Words: - DiffServ, Adaptive, AQM, Robustness, RIO

\section{Introduction}

RIO (RED with IN and OUT)[1] is the primary Active Queue Management (AQM)[2] mechanism used in DiffServ (Differentiated Service) networks. In the core router of AF (Assured Forwarding) Service[3], RIO is adopted to differentiate the quality of incoming flow according to the service class and IN/OUT tags marked by edge router. RIO can be viewed as the combination of two instances of RED[4] for IN and OUT packets respectively. By dropping OUT packet in advance when average queue length increases to dangerous threshold, RIO can protect IN packets from loss.

However, it is difficult to configure RED [5][6] because of its instable performance caused by parameter setting sensitivity. This problem is also named as the robustness problem in [7], which means the RED queues with same parameter settings will have different performance under different traffic load and network condition.

As a variation of RED, RIO also has similar robustness problem, which will be shown in the simulation in Section 2. The queue performance in delay and loss of RIO fluctuate in a large degree under different parameter settings and traffic load. This phenomenon can be explained in following reasons: The throughput of IN queue is always guaranteed high on the cost of dropping OUT packets. Due to the interaction of IN/OUT packet in the same physical queue, the loss of OUT packet cause large fluctuation in delay and loss of the whole queue, which will result in the performance decrease in both IN and OUT queue.

With more and more multimedia application deployed over Internet, it is necessary to provide not only the quality of service in throughput but also in delay and loss. Thus it is important to eliminate the negative effect caused by robustness problem of RIO in DiffServ networks. In this paper, we analyze the steady queue length of RIO in under-subscribed link and investigate its changing principles under different traffic load. We observed that, it is necessary to dynamically adjust the control policy of RIO to keep the queue in stable state. Two adaptive versions of RIO are proposed to solve this problem in delay-sensitive and loss-sensitive application respectively.

The remainder of this paper is organized as follows: Section 2 illustrates the robustness problem of RIO in simulation. Section 3 analyze the queue performance of RIO and discuss the factors affecting robustness of RIO. Section 4 proposes two kinds of adaptive RIO (named by ARIO-1 and ARIO-2 respectively). Section 5 provides the comparison results between original and adaptive version of RIO under dynamic traffic load and different subscription. Finally, Section 6 concludes this paper.

\section{Robustness Problem of RIO}

Before delving into details of the analysis and improvement of robustness of RIO, we first review some simulation results illustrating RIO's sensitivity to parameters, and showing the adaptive version proposed can indeed address this problem.

Fig. 1 and Fig.2 show a set of simulation with a single congested link in a dumbbell topology of DiffServ networks, with the number of long-lived 
TCP flows ranging from 50 to 150 and the subscription ratio of the link ranging from $40 \%$ to $80 \%$. The congested link is $15 \mathrm{Mbps}$ and there are 20 random web flows as background traffic. In Fig.1, the simulation use RIO with $\left(t h_{\min }^{\text {out }}, t h_{\max }^{\text {out }}, p_{\max }^{\text {out }}\right.$ ) and $\left(t h_{\min }{ }^{i n}, t h_{\max }{ }^{\text {in }}, p_{\max }{ }^{\text {in }}\right)$ in value of $(100,600$, $\left.p_{\max }{ }^{\text {out }}\right)$ and $(600,800,0.01) . p_{\max }^{\text {out }}$ changed from 0.01 to 0.2 in each run.

Fig.1(a) shows the tradeoff between utilization of IN queue and the average queue length, while Fig.1 (b) indicates the tradeoff between total loss ratio and the average queue length. Each line presents the results from the simulations with different flows and subscription condition. As shown in figures, although the throughput of IN queue (or utilization of subscribed bandwidth) in RIO queue are kept high above 95\%, the average queue lengths range greatly from 100 to 550, which result in the fluctuation of queue delay from $53.3 \mathrm{~ms}$ to $293.3 \mathrm{~ms}$ with the TCP packet size $1 \mathrm{~K}$ Bytes. The total loss almost increase $2 \%$ for every more 50 flows. With the fixed parameter $p_{\max }$ out , the queue performances in delay and loss of RIO vary greatly against to different traffic load and parameter settings.

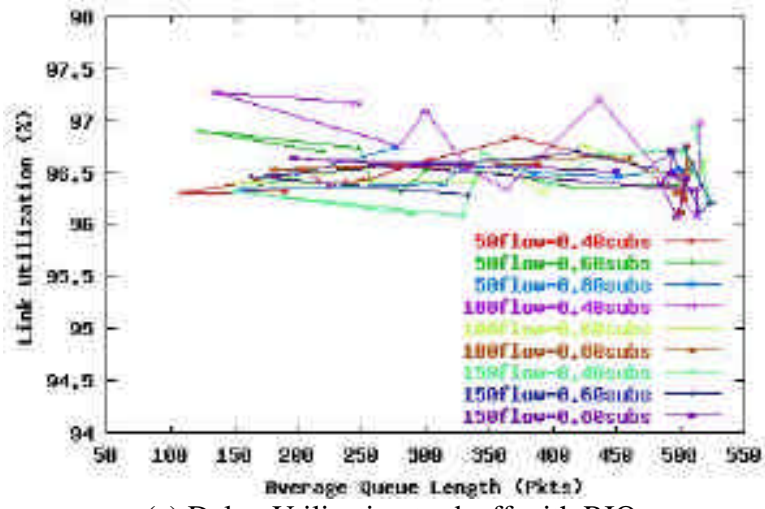

(a) Delay-Utilization tradeoff with RIO

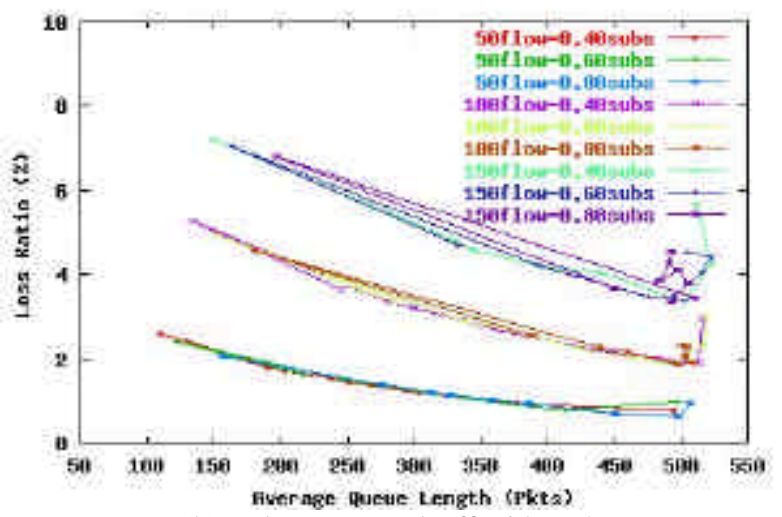

(b) Delay-Loss tradeoff with RIO

Fig.1. Parameter sensitivity of RIO

Fig.2 shows the results of ARIO-1 (one of the two proposed algorithm in Section 4) with the same traffic condition and parameter settings of Fig.1. As shown in figures, by keeping the IN throughput as high as original RIO, the fluctuation of average queue lengths is limited in the small range of 300 to 400 packets, and the total loss ratio are also decreased apparently. The queue performance in delay is in stable value under different traffic load and subscription.

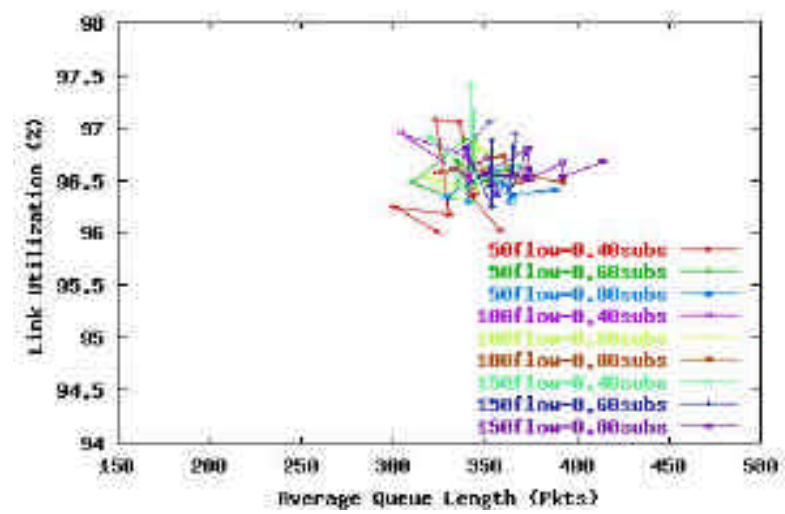

(a) Delay-Utilization tradeoff with ARIO-1

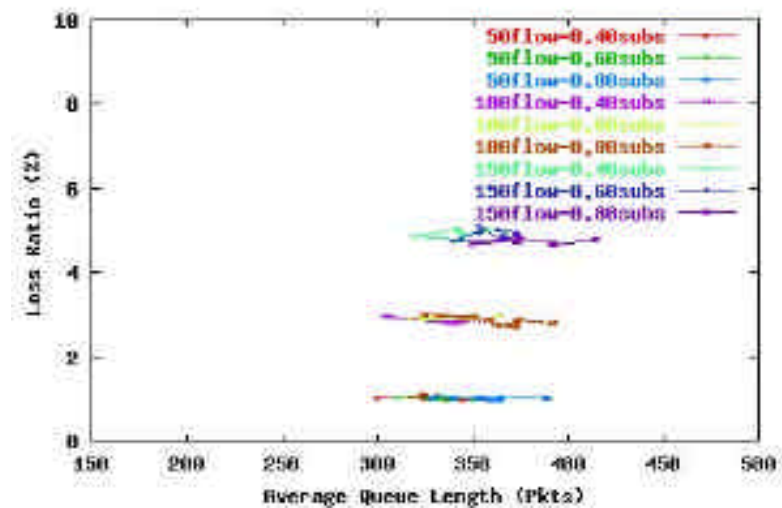

(b) Delay-Loss tradeoff with ARIO-1

Fig.2. Parameter sensitivity of ARIO-1

The set of simulations illustrate that, by adopting proper control policy in RIO, we can get more stable and higher queue performance in delay and loss with different initial parameter, or in another word, increase the robustness of RIO in RIO.

\section{Analysis on the steady queue perf- ormance of RIO}

In this section, we model and analyze the steady queue performance of RIO under different traffic load and user-defined subscription so as to find out the proper RIO control policy for robustness.

\subsection{Steady queue length of TCP aggregates}

In DiffServ architecture, different traffic flows with the same service class will be aggregated into TCP aggregates. In most cases, these aggregates will be marked into IN or OUT by proportional marking algorithm, such as TSW (Time Sliding Window).

Taking the TCP-RIO system in core routers in under-subscribed DiffServ link as our research object, we can get the throughput of an TCP 
aggregate with $n$ flows [8]:

$$
B_{A}=\frac{3}{4} \cdot S+\frac{3}{4} \cdot k \cdot \sum_{i=1}^{n} \frac{1}{R T T_{i}} \sqrt{\frac{2}{p_{i}}}
$$

where $B_{A}$ is the throughput of TCP aggregate, $S$ is the subscription of the link, $k$ is the TCP packet size, $R T T_{i}$ is the average round trip time of $i$ th TCP flow, $p_{i}$ is the average loss ratio of OUT packets in $i$ th flow.

We assume the TCP flows in aggregate having the same $R T T_{i}$ and $p_{i}$ with the value $R T T$ and $p$, then (1) can be rewritten as:

$$
B_{A}=\frac{3}{4} \cdot S+\frac{3}{4} \cdot \frac{k \cdot n}{R T T} \sqrt{\frac{2}{p}}
$$

Assuming ideal proportional marking in edge router and full utilization $\left(B_{A}=C\right)$ in core router, we can get:

$$
p_{m}=\frac{S}{B_{A}}=\frac{S}{C}
$$

On the other side, $R T T$ consist of transmission $R$ delay and queuing delay:

$$
R T T=R+\frac{q}{B_{A}}
$$

where $q$ is the steady average queue length of whole RIO queue.

From (2)-(4), we can get conclusion as follows:

$$
q=\frac{k \cdot n}{4 / 3-S / C} \cdot \sqrt{\frac{2}{p}}-C \cdot R
$$

Thus, the main factors affecting the steady average queue length $q$ include the traffic load or the number of flows $(n)$, the marking probability $\left(p_{m}\right)$ or the subscription $\operatorname{ratio}(S / C)$. The main method to control the changes of $q$ is to change the average loss ratio of OUT packet $(p)$.

\subsection{Simulation Verification}

To verify the analysis conclusion of (5), we run several simulations to investigate the change principles of steady average queue length against to $n$ and $S / C$. In order to observe the steady loss ratio value of OUT packet, we replace the RIO module with fixed dropping probability RIO module, Similar method has been adopted to investigate queue characteristics of RED in [9], and the relationship between $q$ and $p$ was named as Queue Law.

In the first group of simulation, the subscription ratio is kept as $40 \%$, while the number of TCP flows increase from 50 to 300 step by step. Getting rid of the overflow cases, we record the total average queue length and average loss ratio of OUT packets.
The queue law curves are shown in Fig.3(a). The queue law moves towards up-right direction with the increase of the number of flows $n$. The moving step is approximately constant, which implies $q$ has linear relationship with $n$.

In the second group of simulation, the number of flow is kept as 100 , while the subscription ratio increase from $10 \%$ to $80 \%$ step by step. We draw out the queue law curve in the same way with the first group. As shown in Fig.3(b), the queue law moves towards up-right direction with the increase of the subscription degree $S / C$. The moving step of queue law is increasing with $S / C$, which implies the approximately reciprocal relationship between $q$ and $S / C$.

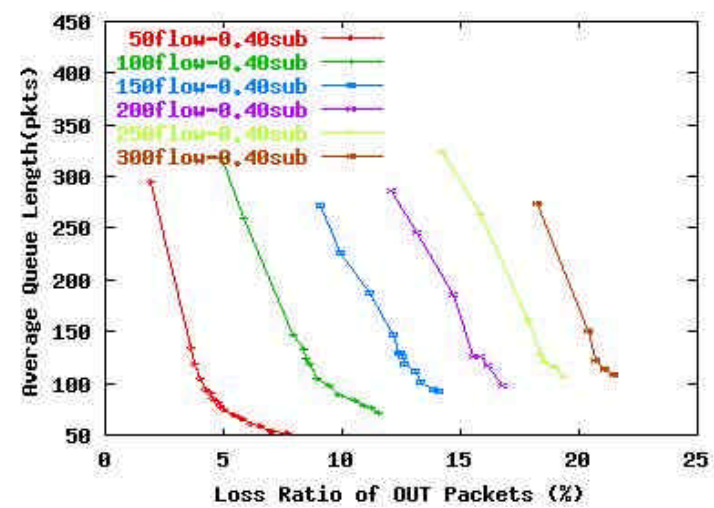

(a) Queue Law under different TCP Load

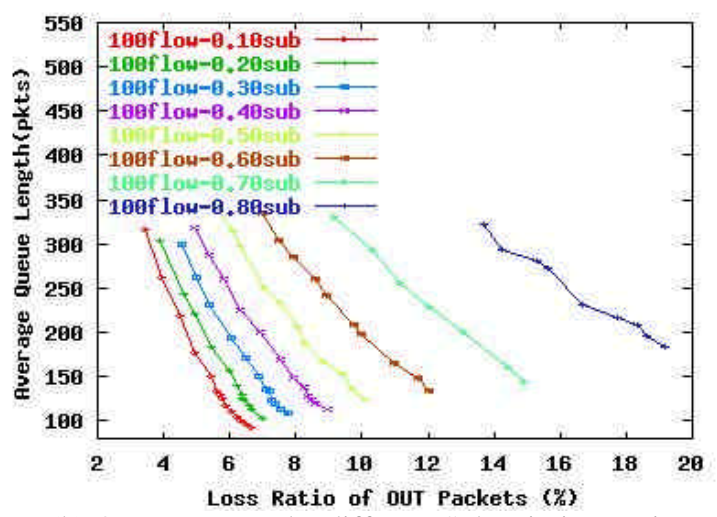

(b) Queue Law under different Subscription Ratio

Fig.3 Queue Law of RIO under dynamic condition

The results above verify the conclusion of (5) that steady queue length of RIO is affected greatly by the number of flows $n$ and the subscription degree $S / C$. It also illustrates the changing principles of steady queue length. In order to eliminate the negative effect of traffic fluctuation, it is necessary to use proper control policy to dynamically adjust the average loss ratio of OUT packet $(p)$.

\section{Adaptive RIO Algorithms}

4.1 Control policy of RIO

In the early work of V.Firoju [9], a control policy 
for stability of RED is proposed as shown in Fig.4. $\boldsymbol{G}(\boldsymbol{p})$ is the average queue length function of loss probability, indicated as Queue Law in the Fig. $\boldsymbol{H}(\boldsymbol{q})$ is RED dropping probability function of average queue length, named as Control Law. The control policy is to make the two law curve have a point of intersection $\left(p_{s}, q_{s}\right)$ in the random dropping stage $\left(0<p_{s}<p_{\max }, t h_{\min }<q_{s}<t h_{\max }\right)$. The intersection point $\left(p_{s}, q_{s}\right)$ is proved to be an equilibrium point and an attracter, which means once the system reaches this point, it will stay there with small fluctuations, given the network traffic load does not changed.

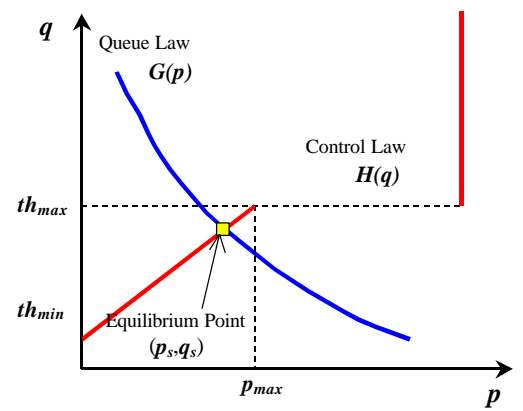

Fig.4 Stable RED Queue System

In the case of under subscription in DiffServ networks, since no IN packets are dropped, the RIO works just in the OUT queue RED part. Thus the stable condition above will also hold true. Based on this idea, two possible adaptive schemes are proposed to adjust the control law curve in Fig.5.

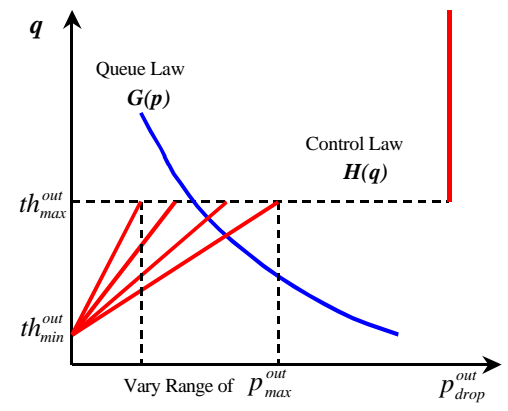

(a) Adjust $p_{\max }^{\text {out }}$

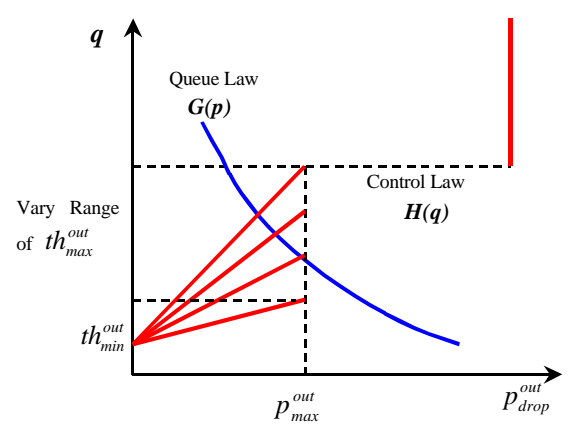

(b) Adjust $t h_{\max }^{\text {out }}$

Fig.5. Dynamically Adjust RIO Control Law

The main idea of the first scheme in Fig.5(a) is keeping the $t h_{\max }{ }^{\text {out }}$ as constant and dynamically adjust $p_{\max }{ }^{\text {out }}$ meet the intersection. This scheme takes specific queue length as control target. Thus it can ensure the QoS of queuing delay and fits for delay-sensitive or jitter-sensitive applications. It is also the essential idea of Adaptive RED[10][7]. The RIO algorithm adopting this scheme is named as ARIO-1.

The idea of the second scheme in Fig.5(b) is to adjust control law in another direction, that is, keeping the $p_{\max }^{\text {out }}$ as constant and dynamically adjust $t h_{\max }{ }^{\text {out }}$ to meet the intersection. This scheme takes specific average loss probability as control target. Thus it can ensure the QoS of loss probability and fits for loss-sensitive applications. The RIO algorithm adopting this scheme is named as ARIO-2.

The essence idea of the two schemes is to make compromise between queuing delay and loss ratio. The improvement of one performance is on the cost of the other.

\subsection{Adaptive RIO Algorithm}

The adaptive control policy of RIO system can be implemented on the basis of original RIO system. In every adaptive period, the change trend of queue length or loss ratio in RIO is estimated by EWMA (Exponentially Weighted Moving Average) method. Additional controller will compare the estimated values with pre-defined performance specification, and then adjust maximum dropping probability $\left(p_{\max }{ }^{\text {out }}\right)$ or maximum queue threshold $\left(t h_{\max }^{\text {out }}\right)$ in OUT queue of RIO.

The two algorithms are given in Fig.6. Considering the value range of $p_{\max }^{\text {out }}$ is $(0,1)$ and multiplicative decrease is helpful to make $p_{\max }$ out approach to 0, the adjustment part of ARIO-1 adopts AIMD (Additive Increase Multiplicative Decrease) method. While the adjustment of ARIO-2 adopts AIAD (Additive Increase Additive Decrease), because the value range of $t h_{\max }^{\text {out }}$ is widely and additive increase/decrease can cover the whole range evenly. Some settings of the fixed parameters take reference of [7].

\section{Simulation and Result}

\subsection{Simulation configuration}

In this section, we design a set of simulations to compare the transient and average performance of the original RIO and two proposed adaptive RIO algorithms in ns-2[11]. 


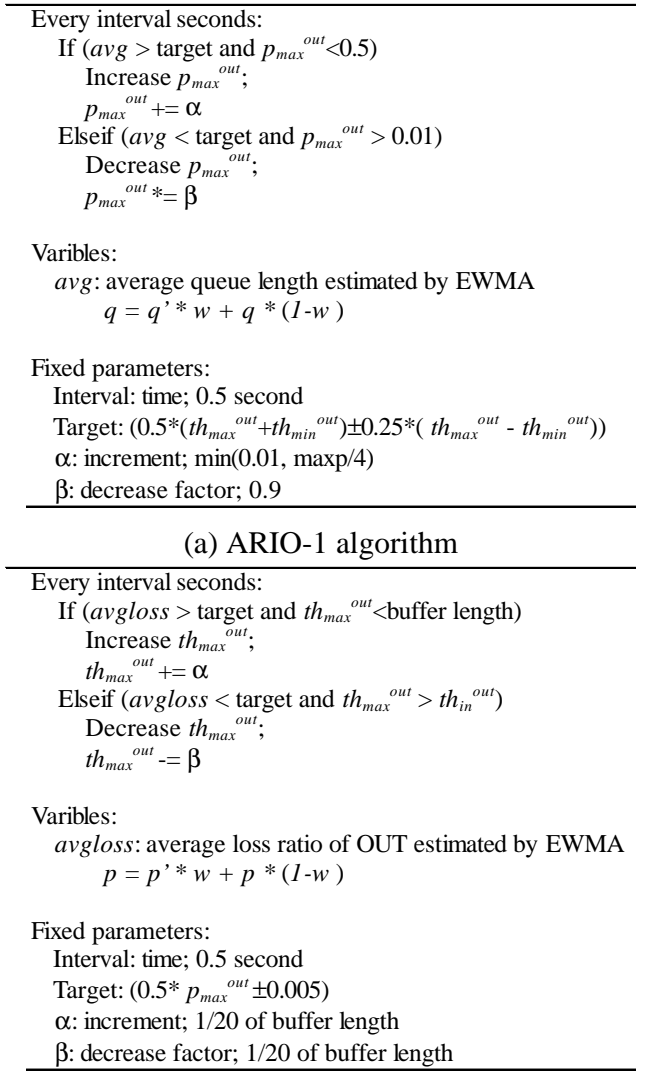

(b) ARIO-2 algorithm

Fig.6. The algorithms for ARIO-1 and ARIO-2

The simulation topology is shown in Fig.7. In a dumbbell link of a DiffServ domain, "edge1" and "edge2" are edge node, and "core" is core node. N TCP connections are created from "src1"-"srcN" to "dst1"-"dstN", each sending FTP bulk-data. TSW maker is installed on the output interface of edge1. The bottleneck link is between core to edge2, with bandwidth of $15 \mathrm{Mbps}$ and transmission delay of $20 \mathrm{~ms}$. The buffer size of core is 1000 packets. All of the other links have high bandwidth of 100Mpbs and low transmission delay of 10ms. The TCP round trip time is $100 \mathrm{~ms}$ approximately and the TCP packet size is $1 \mathrm{~KB}$.

In order to investigate complex varying traffic background, we use the traffic load (the number of TCP flows) shown in Fig.8 to imitate the dynamic TCP aggregates, and change the subscription ratio from $50 \%$ to $90 \%$ in different runs. 40 short random TCP flows are deployed in simulation to act as background Web traffic.

We install the RIO, ARIO-1 and ARIO-2 module on the output interface of core node. The main configurations are list in Table 1.

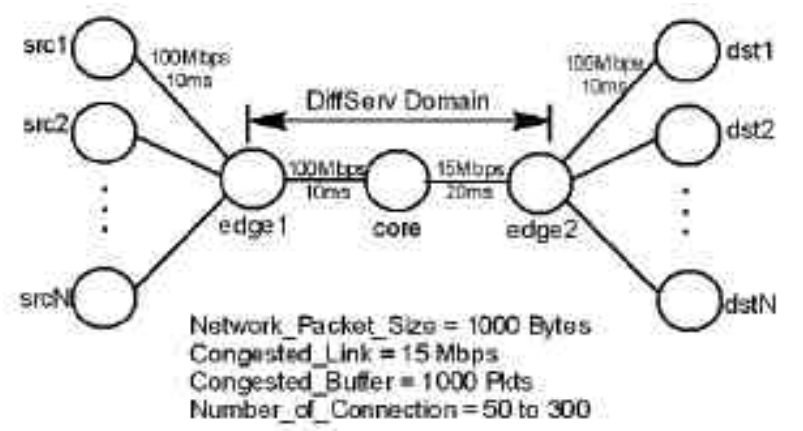

Fig.7 Network Topology in Simulation

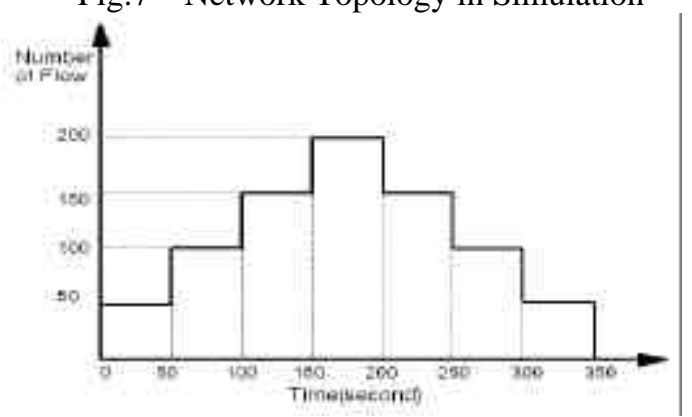

Fig.8 TCP Load (Number of Flow) in Simulation

Table 1. Simulation Configuration

\begin{tabular}{|c|c|c|c|}
\hline Settings & RIO & ARIO-1 & ARIO-2 \\
\hline Buffer Size (pkts) & 800 & 800 & 1500 \\
\hline$\left(\operatorname{th}_{\min }{ }^{\text {out }},{ }^{2}\right.$ h $\left._{\max }{ }^{\text {out }},{ }_{\text {max }}^{\text {out }}\right)$ & $(100,600,0.10)$ & $(100,600,0.10)$ & $(100,600, p)$ \\
\hline$\left(t h_{\min }{ }^{\text {in }}, t h_{\max }{ }^{\text {in }}, p_{\max }{ }^{\text {in }}\right)$ & $(600,800,0.01)$ & $(600,800,0.01)$ & $(600,800,0.01)$ \\
\hline Adaptive parameter & -- & $p_{\max }^{\text {out }}$ & $t h_{\max }^{\text {out }}$ \\
\hline Adaptive period (s) & -- & 0.5 & 0.5 \\
\hline Adaptive Range & -- & $(0.01,0.50)$ & $(100,1000)$ \\
\hline Control target & -- & $1 / 2^{*}\left(t h_{\min }{ }^{\text {out }}+t h_{\max }{ }^{\text {out }}\right)$ & $1 / 2 * p_{\max }{ }^{\text {out }}$ \\
\hline Adjust Parameter & -- & $\alpha=0.1, \beta=0.9$ & $\alpha=\beta=10$ \\
\hline
\end{tabular}

\subsection{Comparison in transient performance}

We compare the transient average length between RIO and ARIO-1 and the transient average loss ratio between RIO and ARIO-2. For space limited, we only show the simulation results in case of $60 \%$ subscription in Fig.9 and Fig.10.

As shown in Fig.9(a), the average queue length of RIO varies linearly with the changes of traffic load in Fig.8. While in Fig9(b), ARIO-1 can effectively keep the steady average queue length around the control target in the middle of OUT queue threshold ( $a v g=350 \mathrm{pkts}$ ).

Similar conclusion on transient loss ratio can be drawn in Fig.10. The loss ratio of RIO varies greatly with the changes of traffic load, while the adaptive version ARIO-2 can keep the steady average loss ratio around the target range (avgloss $=2 \%$ ).

\subsection{Comparison in long-term performance}

We investigate the long-term performance metrics of RIO, ARIO-1 and ARIO-2 under the varying traffic load and different subscription. 


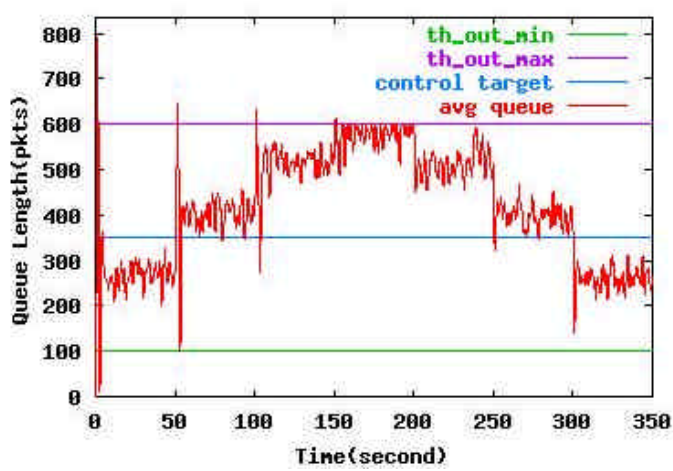

(a) Subscription Ratio $=60 \%$, RIO Algorithm

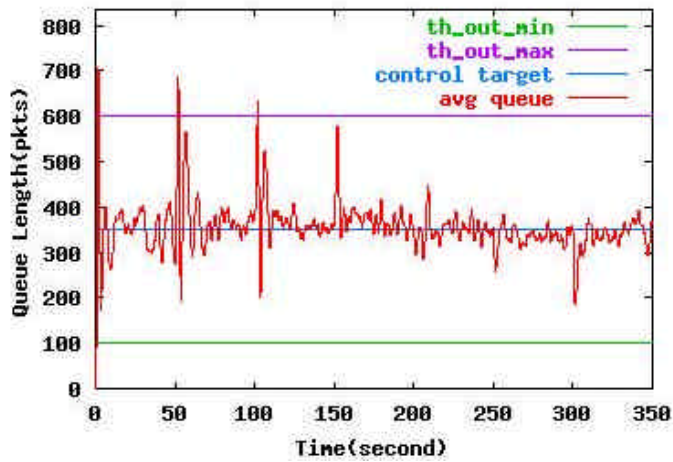

(b) Subscription Ratio $=60 \%$, ARIO-1 Algorithm

Fig.9 Transient performance: average queue length

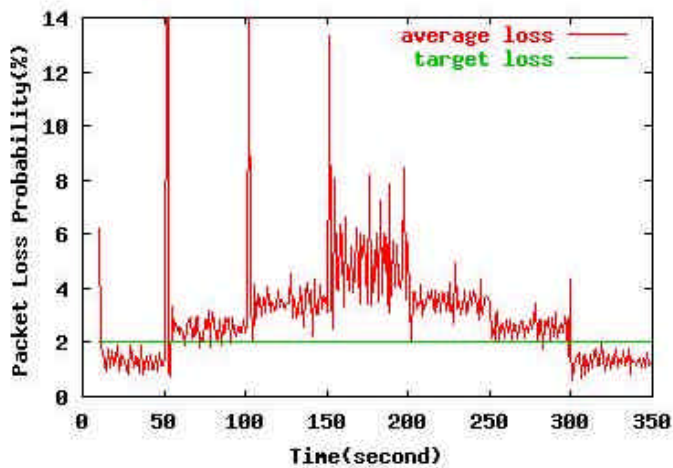

(a) Subscription Ratio $=60 \%$, RIO Algorithm

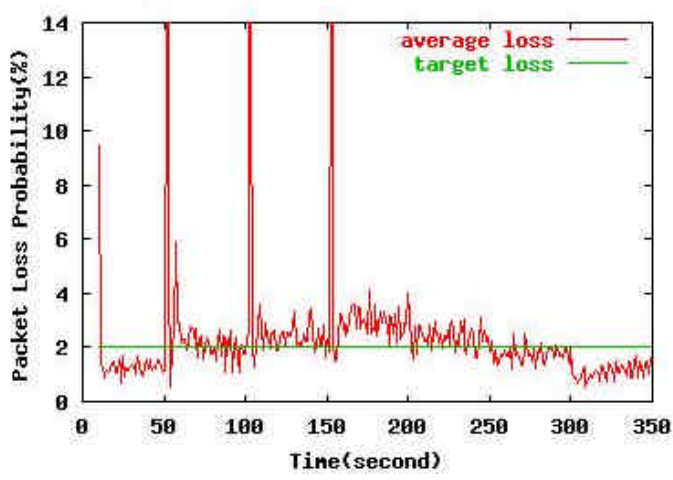

(b) Subscription Ratio=60\%, ARIO-2 Algorithm

Fig.10 Transient performance: average loss ratio

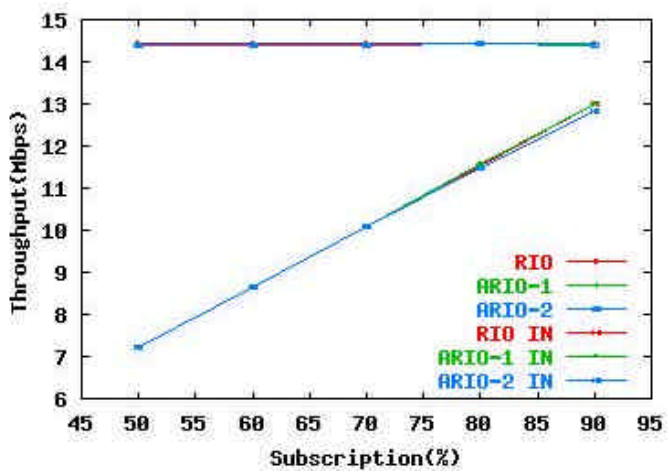

(a) Throughout vs. subscription

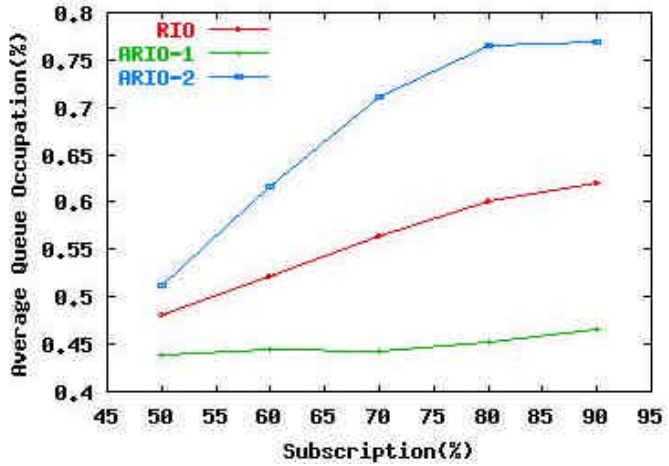

(b) Average queue occupation vs. subscription

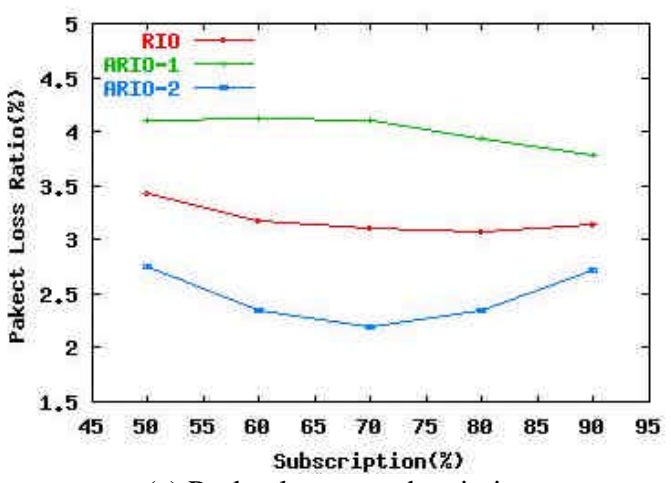

(c) Packet loss vs. subscription

Fig.11 Long-term performance

In Fig.11(a), the long-term total and IN throughput of the three AQMs under varying traffic load and different subscription are almost in the same values, which means the high throughput property of RIO is also held by the two adaptive versions.

Combining the results shown in Fig.11(b) and (c), we can find out the performance benefit of adaptive version in queue delay or loss ratio is always on the cost of the other one. ARIO- 1 can get steady queue occupancy(at 45\%) lower than that of RIO, but it has steady loss ratio(at $4 \%$ ) higher than that of RIO. Similarly, ARIO-2 can get lower loss ratio than RIO but on the cost of high value of queuing delay.

Compared with original RIO, ARIO-1 and ARIO-2 can get more steady performance in delay and loss respectively, which is prerequisite for delay-sensitive and loss-sensitive multimedia 
application over Internet.

\section{Conclusion}

RIO has problems in parameter sensitivity. Although it can always keep high guaranteed throughout in IN queue, the performance of delay and loss of RIO with fixed parameter settings will be undesirable under varying traffic load. With the deployment of multimedia application over Internet, it is not enough to only guarantee throughput. This paper addresses on the issue of robustness of RIO.

As analyzed in this paper, the main factors affecting robustness of RIO include the traffic load and subscription degree. In order to eliminate the negative effect of traffic fluctuation, it is necessary to use proper control policy to dynamically adjust the average loss ratio of OUT packet.

Two adaptive versions of RIO algorithm are proposed and implemented for delay-sensitive and loss-sensitive application respectively. Simulation results show that these two enhanced RIO algorithms can effectively achieve and maintain the control target of queue delay and loss ratio. Without much loss in the guaranteed throughout of IN queue, they increase the robustness of RIO in performance in queue delay and loss ratio.

\section{References:}

[1] D.D.Clark and W.Fang, "Explicit allocation of best-effort packet delivery service", IEEE/ACM Trans. on Networking, Vol.6, No.4,pp.362-373, August 1998.

[2] B.Braden, D.Clark, etc. "Recommendations on Queue Management and Congestion Avoidance in the Internet", RFC 2309, April 1998.

[3] J.Heinanen, F.Baker, W.Weiss, and J.
Wroclawski, "Assured Forwarding PHB Group", Internet RFC 2597, June 1999.

[4] S.Floyd, V.Jacobson, "Random Early Detection Gateways for Congestion Avoidance", IEEE/ACM Trans. on Networking, Auguest, 1993.

[5] T.Ziegler, C. Brandauer, S. Fdida, "A quantitative model for the parameter setting of RED with TCP traffic", in Proc. of Ninth International Workshop on Quality of Service (IWQoS 2001). Karlsruhe, Germany, June 6-8, 2001

[6] K.Chandrayana, B. Sikdar, S. Kalyanaraman, "Scalable configuration of RED queue parameters", in Proc. of 2001 IEEE Workshop on High Performance Switching and Routing, 29-31 May 2001.

[7] S.Floyd, R.Gummadi and S.Shenker, "Adaptive RED: An Algorithm for Increasing the Robustness of RED's Active Queue Management.", http://www.icir.org/ floyd/ red. html, August, 2001.

[8] I.Yeom and A.L.N. Reddy, "Modeling TCP behavior in a Differentiated Services Network", in IEEE/ACM Trans. on Networking, vol.9, no.1, Feb, 2001.

[9] V.Firoiu and M.Borden, "A Study of Active Queue Management for Congestion Control", in Proc. of IEEE INFOCOM'O0, 2000.

[10] W.Feng, D.Kandlur. etc. "A Self-Configuring RED Gateway", in Proc. of IEEE INFOCOM'99, 1999.

[11] VINT Project, "Network Simulator version 2(ns-2)", http://www.isi. edu/nsnam/ns 